\title{
V. On the forcing of oscillations by disturbances of different frequencies
}

\section{Andrew Stephenson}

To cite this article: Andrew Stephenson (1907) V. On the forcing of oscillations by disturbances of different frequencies, Philosophical Magazine Series 6, 14:79, 115-122, DOI: $10.1080 / 14786440709463663$

To link to this article: http://dx.doi.org/10.1080/14786440709463663

曲 Published online: 16 Apr 2009.

Submit your article to this journal $\sqsubset \pi$

山 Article views: 12

Q View related articles $\square$

Citing articles: 1 View citing articles 5 
normally from its end faces-an assumption far from being realized in our actual experiments. Hence the importance of this term must be reduced, when applied to the case usually subject to experiments.

Thus far, the agreement between theory and experiment is in general to be considered as fairly good, if we consider the difficulties encountered in measuring the minute strains caused by magnetization, and also the considerable dependence of the magnetization upon the order of magnetizing and straining. Since the theories, which are based upon quite different considerations, all agree with one another in the first important term, it may be concluded that for the first approximation, they are all verified by the present experiments. It seems, however, impossible to decide experimentally the correctness of the terms of second importance for ferromagnetic substances, in which the hysteresis effect appears in no inconsiderable amount.

In conclusion, we wish to express our best thanks to Dr. $\mathrm{S}$. Sano for useful suggestions in working out the theoretical part of the present investigation.

V. On the Forcing of Oscillations by Disturlances of Different Frequencies. By Andrew Stephenson *.

1. THE simple oscillation of a system may be influenced in two distinct ways : either by a force which is a function of the time alone, or by a force which is a function of both the time and the configuration of the system. In the former case resonance occurs only when the period of the force is equal to the free period of the system; in the latter the effect of the disturbance is cumulative when the ratio of its period to that of the system has any value within a certain range in the vicinity of $\frac{1}{2} r$, where $r$ is any integer + , the intensity being maximum when $r=1$. We now enquire as to the effect of the two different types in joint action. Will the two together under any circumstances produce and continually intensify an oscillation when each acting separately would have no appreciable result?

* Communicated by the Author.

$\dagger$ "On a class of forced oscillations," Quarterly Journal of Pure and Applied Mathematics, no. 148 (1906). The case of double frequency, $\gamma=1$, was examined by Lord Rayleigh, "On the maintenance of vibrations by forces of double frequency, and on the propagation of waves through a medium endowed with periodic structure," Phil. Mag. vol. xxiv. (188\%). 
2. The equation of motion under such action is

$$
\ddot{x}+\mu^{2}\left(1+2 \alpha_{1} \cos n t+2 \alpha_{2} \cos 2 n t+\ldots\right) x=a \sin (q t+\epsilon) \text {. }
$$

The properties of the forced motion follow readily from consideration of the free motion, the solution for which we shall therefore briefly recapitulate ${ }^{*}$. For the free motion, i. e. $a=0$, we have

where

$$
x=\sum_{-\infty}^{\infty} A_{r} \sin \left\{(c-r n) t+\epsilon^{\prime}\right\}
$$

$A_{r}\left\{\mu^{2}-(c-r n)^{2}\right\}+\mu^{2}\left\{\alpha_{1}\left(A_{r-1}+A_{r+1}\right)+\alpha_{2}\left(A_{r-2}+A_{r+2}\right)+\ldots\right\}=0$.

On eliminating the $A$ 's, we obtain the infinite determinant

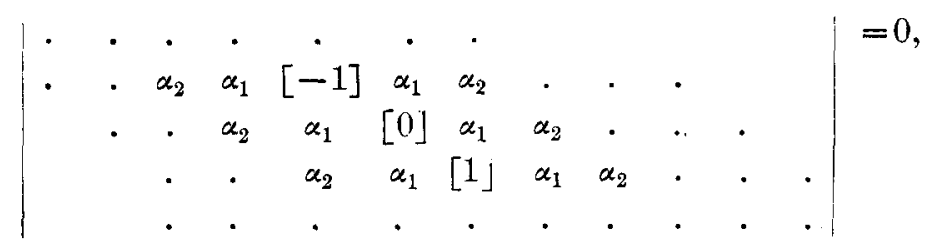

where $[r]$ denotes $\mu^{2}-(c-r n)^{2}$. This equation determines $c$, and the roots are all included in the form $\pm c_{0}-r n$. If $c$ is real the motion is made up of simple elements of constant amplitude, but if $c$ is complex the amplitudes continually increase, as one part of the solution contains a factor $e^{\lambda t}$, where $\lambda$ is a positive quantity. 'The forced motion due to $a \sin (q t+\epsilon)$ is given by

where

$$
x=\sum_{-\infty}^{\infty} \mathrm{A}_{r} \sin \{(q-m) t+\epsilon\},
$$

$\mathrm{A}_{r}\left\{\mu^{2}-(q-r n)^{2}\right\}+\mu^{2}\left\{\alpha_{1}\left(\mathrm{~A}_{r-1}+\mathrm{A}_{r+1}\right)+\alpha_{2}\left(\mathrm{~A}_{r-2}+\mathrm{A}_{r+2}\right)+\ldots\right\}=0$,

except in the case $r=0$, when

$$
\mathrm{A}_{0}\left(\mu^{2}-q^{2}\right)+\mu^{2}\left\{\alpha_{1}\left(\mathrm{~A}_{-1}+\mathrm{A}_{1}\right)+\alpha_{2}\left(\mathrm{~A}_{-2}+\mathrm{A}_{2}\right)+\ldots\right\}=a .
$$

These equations determine a convergent series whatever the value of $\alpha$. The conditional equation $(r)^{\prime}$ is the same as the corresponding equation $(r)$ in the free motion with $q$ for $c$, except that in $(0)^{\prime}$ the right-hand side is a instead of zero. If, therefore, $c$ is real, the $A$ 's become large without limit as $\dot{q}$ is taken nearer and nearer to $c$; and in the limit when $q=c$ the solution passes over into the form

$$
x=t \Sigma \mathrm{B}_{r} \cos \{(q-r n) t+\epsilon\}+\Sigma \mathrm{C}_{\boldsymbol{r}} \sin \{(q-r n) t+\epsilon\} .
$$

* The method is due to Gr. W. Hill, "On the Motion of the Lusar perigee," Collected Works, vol. i. The properties of the infinite determinant and the numerical evaluation of $c$ are dealt with therein. 
Oscillations by Disturbances of Different Frequencies. 117

Thus a large oscillation is forced if $q$ is nearly equal to $\pm c_{0}-r n$, and the effect is continually cumulative if $q= \pm c_{0}-r n$; i. e. if the frequency of the forcing disturbance is equal to the frequency of any periodic element in the free motion under variable spring.

If the variable spring itself forces an osciliation $c$ is complex, and therefore whatever the value of $q$ the forcing disturbance cannot belp in the continual intensification of the oscillation, although it may force a motion of amplitude large compared with $a$.

3. In the above it is assumed that the variation in the force of restitution is periodic. If, however, it is made up of two elemenis of incommensurable frequencies,

$$
\ddot{x}+\mu^{2}\left\{1+2 \alpha_{1} \cos \left(n_{1} t+\epsilon_{1}\right)+2 \alpha_{2} \cos \left(n_{2} t+\epsilon_{2}\right)\right\} x=a \sin q t,
$$

and for the free motion

$$
x=\Sigma \Sigma \Lambda_{r, s} \sin \left\{c t+r\left(n_{1} t+\epsilon_{1}\right)+s\left(n_{2} t+\epsilon_{2}\right)\right\},
$$

where the summation includes the zero and all positive and negative integral values of $r$ and of $s$. On substituting for $x$ we find

$$
\mathrm{A}_{r_{1, s}}\left[\mu^{2}-\left(c+r n_{1}+s n_{2}\right)^{2}\right]+\mu^{2}\left\{\alpha_{1}\left(\mathrm{~A}_{r-1, s}+\mathrm{A}_{r+1, s}\right)+\alpha_{2}\left(\mathrm{~A}_{r, 8-1}+\mathrm{A}_{r, s+1}\right)\right\}=0 \text {, }
$$

a system of equations determining a convergent series $: c$ is obtained as before by eliminating the A's, and the roots are all included in the series $\pm c_{0}+m_{1}+s n_{2}$. The examination of the forced motion follows a similar course to that of the preceding case, and we find that the forcing disturbance has a continually cumulative effect if $q= \pm c_{0}+r n_{1}+s n_{2}$, where $r$ and $s$ have the zero or any positive or negative integral values.

4. If the system is subject to kinetic friction, $\ddot{x}+2 k \dot{x}+\mu^{2}\left\{1+2 \alpha_{1} \cos n t+2 \alpha_{2} \cos 2 n t+\ldots\right\} x=a \sin (q t+\epsilon)$.

To find the free motion we put $x=e^{-k t} y$; then

$$
\ddot{y}+\mu^{2}\left\{1-k^{2} / \mu^{2}+2 \alpha_{1} \cos n t+2 \alpha_{2} \cos 2 n t+\ldots\right\} y=\alpha e^{i t} \sin (q t+\epsilon) \text {. }
$$

The complementary function is found as before: it is clear that in all cases, whether the " $c$ " of $y$ is real or complex, $s:$ must in part contain a harmonic series with an exponential function of $t$ as factor. Now the free motion is also given directly by

$$
x=\sum_{-\infty}^{\infty}\left[A_{r} \sin \{(c-r n) t+\epsilon\}+\mathrm{B}_{r} \cos \left\{(c-r n) t+\epsilon_{\}}^{\}}\right],\right.
$$


where

$$
\begin{aligned}
& \mathrm{A}_{r}\left\{\mu^{2}-(c-r n)^{2}\right\}+\mu^{2}\left\{\alpha_{1}\left(\mathrm{~A}_{r-1}+\mathrm{A}_{r+1}\right)\right. \\
& \left.\quad+\alpha_{2}\left(\mathrm{~A}_{r-2}+\mathrm{A}_{r+2}\right)+\ldots\right\}-2 k(c-r n) \mathrm{B}_{r}=0 \quad . \quad\left(r_{1}\right) \\
& \mathrm{B}_{r}\left\{\mu^{2}-(c-r n)^{2}\right\}+\mu^{2}\left\{\alpha_{1}\left(\mathrm{~B}_{r-1}+\mathrm{B}_{r+1}\right)\right. \\
& \left.\quad+\alpha_{2}\left(\mathrm{~B}_{r-2}+\mathrm{B}_{r+2}\right)+\ldots\right\}+2 k(c-r n) \mathrm{A}_{r}=0 . \quad . \quad\left(r_{2}\right\}
\end{aligned}
$$

Comparing the two solutions, we observe that $c$ must be complex. For the forced oscillations we replace $c$ by $q$ and the right-hand side of $(0)^{\prime}$ by $a: c$ being complex, it follows by the argument of $\S 2$, that there is a definite limit to the amplitude of the forced motion due to $a \sin (q t+e)$, although this limit may be large compared with $a$ if the frictional resistance is sufficiently small.

It may be noted that for the numerical evaluation of a forced oscillation under friction, it is expedient to replace the trigonometric functions in the equation of motion by their exponential equivalents and to solve in exponential series.

5 . When the variation in the spring is small, the solution for the forced motion may be obtained readily by approximation. We shall investigate this in the frictionless case, apart from the general theory, thus arriving at a more distinct. conception of how the magnifying effect is produced.

If the equation of motion is

$$
\ddot{x}+\mu^{2}(1+2 \alpha \cos n t) x=\alpha \sin (q t+\epsilon),
$$

for the forced oscillation

$$
x=\sum \mathrm{A}_{r} \sin \{(q-r n) t+\epsilon\}, . . .
$$

where

$$
\mathrm{A}_{r}=a \frac{\left(-\alpha \mu^{2}\right)^{|r|}}{\prod_{0}^{r}\left\{\mu^{2}-(q-r n)^{2}\right\}} \text { approximately, }
$$

provided $\mu^{2}-(q-r n)^{2}$ is not small for any of the values of $r$. In general, therefore, the motion approximates to the forced motion under constant spring. In the exceptional case when $\mu^{2}-(q-r n)^{2}$ is small for some value of $r, m$ say, we consider the solution as made up of two parts, $y$ and $z$, where $y$ is that part of the series in (i) which lies on the same side of the term in $\sin \{(q-m n) t+\epsilon\}$ as the term in $\sin (q t+\epsilon)$. Thus

$$
\ddot{y}+\mu^{2}(1+2 \alpha \cos n t) y=a \sin (q t+\epsilon)+b \sin \{(q-m n) t+\epsilon\} \text {, }
$$

where

$$
b=a \frac{\left(-\alpha \mu^{2}\right)^{m \mid}}{\prod_{0}^{m-\prod^{m \mid}}\left\{\mu^{2}-\left(q-r^{\prime \prime} \mu\right)^{2}\right\}},
$$


Oscillations by Disturbances of Different Frequencies. 119 and therefore

$$
\ddot{z}+\mu^{2}(1+2 \alpha \cos n t) z=-b \sin \{(q-m n) t+\epsilon\} .
$$

On putting $q-m n=\mu-p$, where $p$ is small, we have

in which

$$
z=\sum_{-\infty}^{\infty} \mathrm{B}_{r} \sin \{(\mu-p-r n) t+\epsilon\}
$$

$$
\mathrm{B}_{r}(r n+p)(2 \mu-p-r n)+\alpha \mu^{2}\left(\mathrm{~B}_{r-1}+\mathrm{B}_{r+1}\right)=0,
$$

but for $r=0$,

$$
\mathrm{B}_{0} p(2 \mu-p)+\alpha \mu^{2}\left(\mathrm{~B}_{-1}+\mathrm{B}_{1}\right)=-b \text {. }
$$

In order that $\mathrm{B}_{0}$ may be large compared with $a, p$ must be of order $\alpha^{2}$ and be adjusted correctly to the order $\alpha^{\mid m i+1}$. If $p$ has the value obtained by putting $b=0$ in the conditional equations and eliminating the $\bar{B}$ 's, the solution for the forced oscillations passes over into the form

$$
z=t \sum_{-\infty}^{\infty} \mathrm{B}_{r} \cos \{(\mu-p-r n) t+\epsilon\}+\sum_{-\infty \text { to }-1}^{1 \text { to } \infty} \mathrm{C}_{r} \sin \{(\mu-p-r n) t+\epsilon\} \text {, }
$$

where

and

$$
\mathrm{B}(r n+p)(2 \mu+p-r n)+\alpha \mu^{2}\left(\mathrm{~B}_{r-1}+\mathrm{B}_{r+1}\right)=0
$$

$$
-2 \mathrm{~B}_{r}(\mu-p-r n)+\mathrm{C}_{r}(m+p)(2 \mu-p-m)+\alpha \mu^{2}\left(\mathrm{C}_{r-1}+\mathrm{C}_{r+1}\right)=0,
$$

except for $r=0$, when

Hence

$$
-2 \mathrm{~B}_{0}(\mu-p)+\alpha \mu^{2}\left(\mathrm{C}_{-1}+\mathrm{C}_{1}\right)=-b .
$$

$$
\mathrm{B}_{0}=+b / 2 \mu, \text { and } \mathrm{B}_{r}=\mathrm{B}_{0} \frac{\left(-\alpha \mu^{2}\right)^{|r|}}{\prod_{1}^{r} r n(2 \mu-r n)} \text { approximately. }
$$

Thus the rate of increase of amplitude is proportional to $\mid \alpha^{m}$, a result which shows how rapidly the intensity of the magnifying effect diminishes as $m$ increases.

In practice, the equation of motion is not linear in $x$, the force of restitution being in general an odd function of $x$ containing $x^{3}$ and higher powers ; nevertheless the above investigation bolds good so long as $x^{3}$ is negligible compared with $b, i . e$. if $a^{2}$ is small compared with

$$
\left(\alpha \mu^{2}\right)^{|m|} \prod_{1}^{m-\frac{\mid m i}{m i}}\left\{\mu^{2}-(q-r n)^{2}\right\} .
$$

If this condition is not satisfied the linear equation does not furnish a sound approximation, and higher powers of $x$ 
must be included. The magnifying effect of the joint disturbance depends upon the adjustment of the frequencies ; and as the frequency of the free motion is a function of the amplitude, the action can have appreciable effect in any particular case only for a certain range of amplitude.

6. The examination above brings out the interesting fact that when the system is sensitive to the disturbance the oscillation generated is approximately of free period (under variable spring): thus a system of which the spring undergoes periodic variation furnishes, in these cases, an exception to the rule that forced vibrations follow the period of the exciting cause. If a crowd of direct disturbances acts on the system each in general produces a forced oscillation of its own period: but these elements are small in comparison with the oscillations of approximately natural period generated by those disturbances to which the system is sensitive. In this action, therefore, energy is given out by the system in the period in which incident energy is most readily absorbed.

The phenomenon of fluorescence is the outstanding physical case of a direct disturbance exciting an oscillation of different period, and it has been shown that in glowing sodium vapour "the fluorescence spectrum is the exact complement of the absorption spectrum" * Thus an assemblage of simple systems of variable spring furnishes a mechanical analogue to the vapour with regard to fluorescence, at least in so far as the main features are concerned.

It is specially to be noted that the fluorescent spectrum depends upon the frequency of the incident monochromatic light; this is in accordance with our analogy, for of the assemblage of systems only a certain definite group is sensitive to a disturbance of given frequency. From this point of view, again, the lengthening and the shortening of the period of the incident disturbance in emission are essentially similar phenomena, and the exceptions to Stokes' law cease to be exceptional.

7. Owing to the counteracting influence of friction it is difficult to realize more than a few cases of the joint action in practice, but the effect may be observed readily in the two

* "The Fluorescence and Absorption Spectra of Sodium Vapour," by Prof. R. W. Wood and Mr. J. H. Moore; Phil. Mag. Sept. 1903. Prof. Wood found subsequently, Phil. Mag. Nov, 1906, p. 499, on more detailed examination that many absorption lines do not appear in the fluorescent spectrum. In our unalogue these would correspond to vibrations of constant spring. It may be noted that the equidistance of the lines of a group in the fluorescent spectrum produced by monochromatic stimulation is in accordance with the properties of the mechanical system. 
Oscillations by Disturlances of Different Frequencies. 121

leading cases given by $q=|c \pm n|$. A body suspended by a light spring forms a convenient system for this purpose. When the body is set into vibration vertically and the spring seized at the point of suspension and oscillated horizontally, a comparatively large pendulum motion is generated if the sum or difference of the vertical and free pendulum frequencies is approximately equal to the suspension frequency.

In a system with two, or more, degrees of freedom, if one normal coordinate is subject to direct forced oscillation, a variation of frequency $n$, say, is in general produced in the spring of the other coordinate, which will therefore respond to a direct force of frequency $c+r n$.

8. If a system containing a cyclic coordinate is making small oscillations about a state of steady motion under the action of a periodic disturbance, the effect of the disturbance at any instant depends partly upon the configuration of the system in the small oscillations. If only two coordinates are affected the equations for the small oscillations in frictionless motion are of the form

$$
\begin{aligned}
\ddot{x}+a \mu(1 & \left.+2 \alpha_{1} \cos n t+2 \beta_{1} \sin n t+\ldots\right) \dot{y}+\mu^{2}\left(1+2 \alpha_{2} \cos n t\right. \\
& \left.+2 \beta_{2} \sin n t+\ldots\right) x=\mathrm{E} \sin q t+\mathrm{F} \cos q t, \\
\ddot{y}+a^{\prime} \mu^{\prime}\left(1+2 \alpha_{1}^{\prime} \cos n t+2 \beta_{1}^{\prime} \sin n t+\ldots\right) \dot{x}+\mu^{\prime 2}\left(1+2 \alpha_{2}^{\prime} \cos n t\right. & \\
& \left.+2 \beta_{2}^{\prime} \sin n t+\ldots\right) y=\mathrm{E}^{\prime} \sin q t+\mathrm{F}^{\prime} \cos q t,
\end{aligned}
$$

where powers and products of $x$ and $y$ are neglected. The complementary function is of form

$$
\begin{aligned}
& x=\sum_{-\infty}^{\infty}\left[\mathrm{A}_{r} \sin (c-r n) t+\mathrm{B}_{r} \cos (c-r n) t\right], \\
& y=\sum_{-\infty}^{\infty}\left[\mathrm{A}_{r}^{\prime} \sin \left(c-r^{r}\right) t+\mathrm{B}_{r}^{\prime} \cos (c-r n) t\right] .
\end{aligned}
$$

On substituting for $x$ and $y$ and eliminating the coefficients from the conditional equations the frequencies $c_{1}$ and $c_{2}$ are obtained. Complex values of $c$ indicate that the disturbance acting throngh the spring alone produces a cumulative effect. The forced oscillations are of similar form, but with $q$ for $c$; and if $c$ is real it is evident, by the argument previously employed, that the oscillation is of continually increasing amplitude if

$$
q= \pm c_{1}-r n \text { or } \pm c_{2}-r n \text {. }
$$

The approximation made in taking the equations of motion as linear holds good for $r=m$ if the amplitude of the forcing disturbance is small compared with the $m$ th power of that of the spring; otherwise higher powers of $x$ and $y$ must be taken into account in the equation of motion. 
As an example, consider the question of the stability of a symmetrical top spinning in small oscillation about the vertical when acted on by a periodic force through a point in the axis. If the force is vertical the equations of motion are :

$$
\begin{aligned}
-\mathrm{A} \ddot{\eta}+\mathrm{C} m \dot{\xi}+\mathrm{M} g h(1+\alpha \cos n t) \eta & =0, \\
\mathrm{~A} \ddot{\xi}+\mathrm{C} m \dot{\eta}-\mathrm{M} g h(1+\alpha \cos n t) \xi & =0,
\end{aligned}
$$

so that the effect of the applied force, to this degree of approximation, is to produce a variation in the spring alone; hence a comparatively large oscillation is generated if $n$ lies near to a certain value in the vicinity of $2 \mu_{1} / r$ or $2 \mu_{2} / r$, where $\mu_{1}$ and $\mu_{2}$ are the natural frequencies and $r$ any integer. If, on the other hand, the force is horizontal the spring is not affected, and there is instability only when $q=\mu_{1}$ or $\mu_{2}$. When the horizontal and vertical forces act together,

$$
\begin{aligned}
-\mathrm{A} \ddot{\eta}+\mathrm{C} m \dot{\xi}+\mathrm{M} g h(1+\alpha \cos n t) \eta=\mathrm{M} g h a \sin (q t+\epsilon), \\
\mathrm{A} \ddot{\xi}+\mathrm{C} m \dot{\eta}-\mathrm{M} g h(1+\alpha \cos n t) \xi=0 ;
\end{aligned}
$$

so that the joint action produces a large effect when $q=|c \pm r n|$, where $c$ represents either of the frequencies under the vertical force alone.

The spring and direct disturbances may be produced by giving the point of suspension periodic motions in the vertical and horizontal directions respectively; and for readily appreciable magnification it would be necessary to have the vertical vibration of large amplitude.

VI. Diffusion of Gases as an Irreversible Process. By S. H. Burbury, F.R.S.*

DRofessor G. H. Bryan in his recent work on Thermodynamics (Leipzig, Teubner \& Co.) states (p. 125), "when two gases at equal pressure and temperature mix by diffusion, the loss of available energy and consequent gain of entropy is the same as would occur if each component were to expand by rushing into vacuum till it occupied the same volume as the mixture." This statement appears to present some difficulties.

If in a cylinder of volume $2 v$, one half on one side of the piston is occupied by oxygen at given pressure and temperature, and the other half is vacuum, we have in that arrangement available energy. We might, by placing the axis of the cylinder vertical with the oxygen at the bottom,

\footnotetext{
* Communicated by the Author.
} 